\title{
Beaming and Jets in Gamma-Ray Bursts
}

\author{
Re'em Sari \\ Theoretical Astrophysics 130-33, California Institute of Technology, Pasadena CA 91125
}

\begin{abstract}
The origin of GRBs has been a mystery for almost 30 years. The afterglow observed in the last few years enabled redshift determination for a handful of bursts, and the cosmological origin is now firmly established. Though the distancc scalc is settled, there still remains orders of magnitude uncertainty in their rate and in the total energy that is released in the explosion due to the possibility that the emission is not spherical but jet-like. Contrary to the GRB itself, the afterglow can bc measured up to months and even years after the burst, and it can provide crucial information on the geometry of the ejecta. We review the theory of afterglow from jets and discuss the cvidence that at least some of the bursts are not spherical. We discuss the prospects of polarization measurements, and show that this is a powerful tool in constraining the geometry of the explosion.
\end{abstract}

\section{JETS? - A FUNDAMENTAL QUESTION}

The study of $\gamma$-ray bursts was revolutionized when the Italian-Dutch satellite BeppoSAX delivered arcminutes positioning of some GRBs within a few hours after the event. This enabled other ground and space instruments to monitor the relatively narrow error boxes. Emission in X-ray, infrared, optical and radio, so called "afterglow", was observed by now for more than a dozen of bursts.

The current understanding of the GRBs phenomenon is that a compact source emits relativistic flow with Lorentz factor $\gamma$ of at least a few hundreds. This flow emits, probably by internal shocks (see e.g., $[2,3]$ ), the GRB. After these internal shocks have produced the GRB, the ultra relativistic flow interacts with the surrounding medium and decelerates. Synchrotron radiation is emitted by the heated surrounding matter. As more and more of the surrounding mass is accumulated, the flow decelerates and the emission shifts to lower and lower frequencies. Excitingly, the afterglow theory is relatively simple. It deals with the emission on timescales much longer than those of the GRBs. The details of the complex initial conditions are therefore forgotten and the evolution depends only on a small number of parameters.

We begin by clarifying some of the confusing terminology. There are two distinct, but related, effects. The first, "jets", describes scenarios in which the relalivistic flow emitted from the source is not isotropic but collimated in a finite solid angle.

CP526, Gamma-Ray Bursts: $5^{\text {th }}$ Huntsville Symposium, edited by R. M. Kippen, et al. (C) 2000 American Institute of Physics 1-56396-947-5/00/ $\$ 17.00$ 


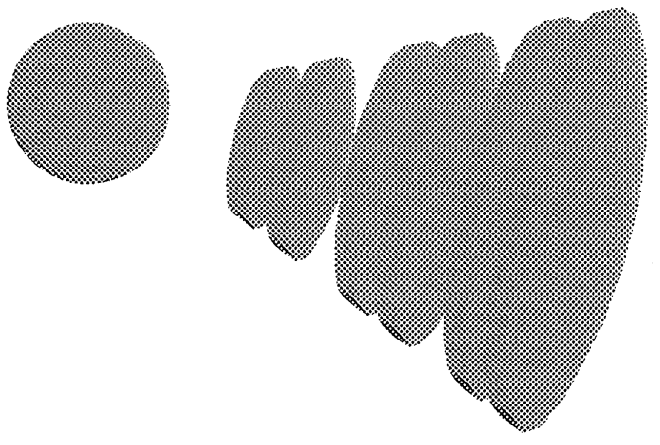

FIGURE 1. Schematic geometric description of jets in GRBs. The scheme shows the multiple shells before internal shocks have occurred. After that they all merge to one shell with typical width a factor of $\gamma^{2}$ thinner than their distance from the source.

The term jet refers to the geometrical shape of the relativistic flow emitted from the inner engine. The second effect is that of "relativistic beaming". The radiation from any object that is radiating isotropically in its own rest frame, but moving with a large Lorentz factor $\gamma$ in the observer frame, is collimated into a small angle $1 / \gamma$ around its direction of motion. This is an effect of special relativity. It has nothing to do with the ejecta's geometry (spherical or jet) but only with the fact that the ejecta is moving relativistically. The effect of relativistic beaming allows an observer to see only a small angular extent, of size $1 / \gamma$ centered around the line of sight. Unfortunately, the term beaming was also used for "jets" by many authors (including myself). We will keep a clear distinction between the two in this paper. Since we know the flow is ultra-relativistic (initially $\gamma>100$ ), there is no question that the relativistic beaming is always relevant for GRBs. The question we are interested in is that of the existence of "jets".

The idealized description of a jet is a flow that occupies only a conical volume with half opening angle $\theta_{0}$. In fact, the relativistic dynamics are such that the width of the matter in the direction of its propagation is much smaller than its distance from the source by a factor of $1 / \gamma^{2}$. The flow, therefore, does not fill the whole cone. Instead it occupies only a thin disk at its base, looking more like a flying pancake [4] - see Figure 1. If the "inner engine" emits two such jets in opposite directions then the total solid angle towards which the flow is emitted is $\Omega=2 \pi \theta_{0}^{2}$. The question whether the relativistic flow is in the form of a jet or a sphere has three important implications.

The Total Emitted Energy. Optical observations of afterglows enabled redshift determination, and therefore a reasonably accurate estimate of the distance, $D$, to these events (the uncertainty is now in the cosmological parameters of the universe). The so called "isotropic energy" can then be inferred from the fluence $F$ (the total observed energy per unit area at earth) as $E_{i s o}=4 \pi D^{2} F$ (taking cosmological corrections into account, $D=D_{L} / \sqrt{1+z}$ where $D_{L}$ is the luminosity distance 
and $z$ is the redshift). The numbers obtained in this way range from $10^{51} \mathrm{erg}$ to $10^{54} \mathrm{erg}$ with the record of $3 \times 10^{54} \mathrm{erg}$ held by the famous GRB 990123. These huge numbers approach the equivalent energy of a solar mass, all emitted in a few tens of seconds!

These calculations assumed that the source emitted the same amount of energy in all directions. If instead the emission is confined to some solid angle $\Omega$, then the true energy is $E=\Omega D^{2} F$. As we show later, $\Omega$ is very weakly constrained by the GRB itself and can be as low as $10^{-6}$. If so, the true energy in each burst is $E \ll E_{i s o}$. We will show later that interpretation of the multi-wavelength afterglow lightcurves indeed indicates that some bursts are jets with solid angles considerably less than $4 \pi$. The isotropic energy estimates may be fooling us by a few orders of magnitudes! Clearly this is of fundamental importance when considering models for the sources of GRBs.

The Event Rate. BATSE sees about one burst per day. With a few redshifts measured this translates to about $10^{-7}$ bursts per year per galaxy. However, if the emission is collimated to $\Omega \ll 4 \pi$ then we do not see most of the events. The true event rate is then larger than that measured by BATSE by a factor of $4 \pi / \Omega$. Again this is of fundamental importance. Clearly, the corrected GRB event rate must not exceed that of compact binary mergers or the birth rate of massive stars if these are to produce the majority of the observed GRBs.

The Physical Ejection Mechanism. Clearly, different physical models are needed to explain collimated and isotropic emission. For example, in the collapsar model (e.g., [1]), relativistic ejecta that is capable of producing a GRB is produced only around the rotation axis of the collapsing star with half opening angle of about $\theta_{0} \cong 0.1$. Such models would have difficulties in explaining isotropic bursts as well as very narrow jets.

With these uncertainties we are therefore left with huge ignorance in how, how much and how many GRBs are produced. The question as to whether the emission of GRBs is spherical or collimated in jets is fundamental to almost all aspects of the GRB phenomenon.

\section{AFTERGLOW SPECTRUM - BASIC THEORY}

When the ejecta interacts with the surrounding medium, a shock wave (the so called the forward shock) is going through the cold ambient medium and heating it up to relativistic temperatures. The basic afterglow model assumes that electrons are accelerated by the shock into a powerlaw distribution of their Lorentz factor $\gamma_{e}: N\left(\gamma_{e}\right) \sim \gamma_{e}^{-p}$ for $\gamma_{e}>\gamma_{m}$. The lower cutoff of this distribution is assumed to be a fixed fraction of equipartition. It is also assumed that a considerable magnetic field is being built behind the shock, again characterized by a certain fraction of equipartition. The relativistic electrons then emit synchrotron radiation and produce the observed afterglow. The broad band spectrum of such emission was given by Sari, Piran \& Narayan [5] (see Figure 2). 

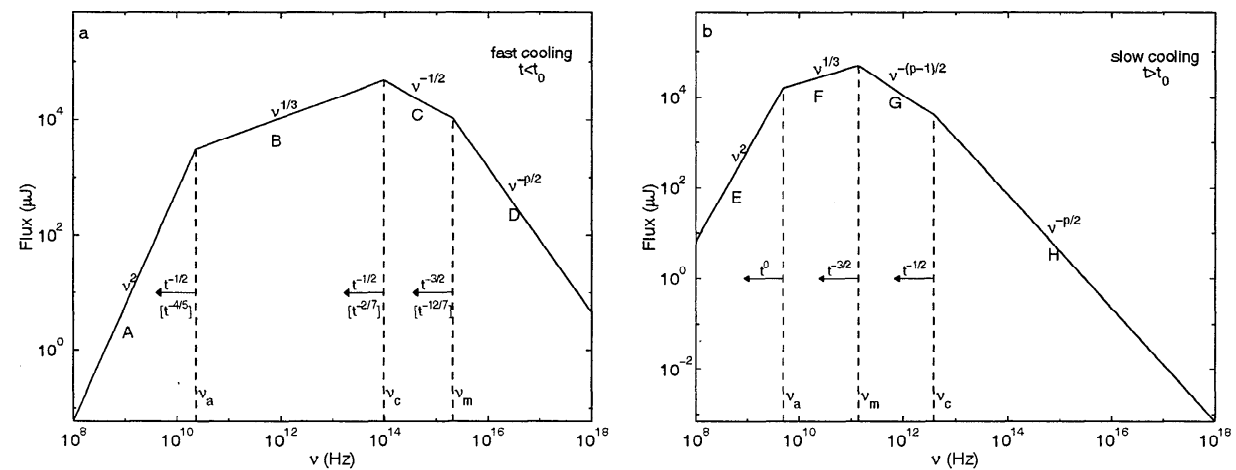

FIGURE 2. Theoretical spectra of synchrotron emission from fast cooling $\left(\nu_{c}<\nu_{m}\right.$ left) and slow cooling $\left(\nu_{m}<\nu_{c}\right.$ right) powcrlaw distribution of electrons. This spectrum is robust and holds for jets as well as spherical ejecta. In general, the break frequencies change in time as well as the overall normalization. The arrows on the figure indicate the evolution of these break frequencies for a spherical emission in a constant density environment. $p=2.2-2.4$ fits the observed spectra well.

At each instant, there are three characteristic frequencies. (i) $\nu_{m}$ is the synchrotron frequency of the minimal energy electron, having a Lorentz factor $\gamma_{m}$. (ii) The cooling time of an electron is inverse proportional to its Lorentz factor $\gamma_{e}$. Therefore, electrons with a Lorentz factor higher than some critical value $\gamma_{e}>\gamma_{c}$ can cool on the dynamical timescale of the system. This characteristic Lorentz factor corresponds to the "cooling frequency" $\nu_{c}$. (iii) Below some crilical frequency $\nu_{a}$ the flux is self absorbed and is given by the Rayleigh-Jeans portion of a black body spectrum. The broad band spectrum of the well studied GRB 970508 [6] is in very good agreement with the theoretical picture.

We stress that the spectrum given above is quite robust. The only assumption is synchrotron radiation from a powerlaw distribution of relativistic electrons. The same spectrum will hold whether the shock propagates into a constant density interstellar medium or a decreasing surrounding density produced earlier by the progenitor's wind. It will be valid whether the ejecta is spherical or jet-like, and whether the equipartition parameters are constant with time or not.

On the contrary, the temporal evolution of the spectrum is more subtle. The simplest evolution, which well describes the data of some bursts, is the spherical adiabatic model with a constant density ambient medium. In this scenario, $\gamma \sim$ $R^{-3 / 2}$, or in terms of the observer time, $t=R / \gamma^{2} c, \gamma \sim t^{-3 / 8}$. Given the evolution of $\gamma(t)$ onc can derive the temporal cvolution of the brcak frcquencics, and the results are indicated in Figure 2. The peak flux in the adiabatic, spherical constant ambient density model is constant with time. 


\section{HYDRODYNAMICS OF JETS}

Interestingly, due to the effect of relativistic beaming (which is independent of jets) we are only able to see an angular extent of $1 / \gamma<0.01$ during the GRB itself where the Lorentz factor $\gamma>100$. Moreover, it is only regions of size $1 / \gamma$ that are causally connected. Therefore, each fluid element evolves as if it is part of a sphere as long as $1 / \gamma<\theta_{0}$. Combining these two facts, we cannot distinguish a jet from spherical ejecta as long as $1 / \gamma<\theta_{0}$.

However, as the afterglow cvolves, $\gamma$ decreases and it will eventually fall below the initial inverse opening angle of the jet. The observer will notice that some of the sphere is missing from the fact that less radiation is observed. This effect alone will produce a significant break, steepening the lightcurve decay by a factor of $\gamma^{2} \sim t^{-3 / 4}$ even if the dynamics of each fluid element has not changed. The transition should occur at the time $t_{\text {jet }}$ when $1 / \gamma \cong \theta_{0}$. Observing this time can therefore provide an estimate of the jet's opening angle according to

$$
t_{\text {jet }} \approx 6.2\left(E_{52} / n_{1}\right)^{1 / 3}\left(\theta_{0} / 0.1\right)^{8 / 3} \mathrm{hr} .
$$

Additionally, Rhoads [7] has shown that at about the same time (see however $[8-10])$, the jet will begin to spread laterally so that its opening angle $\theta(t)^{\prime} \sim 1 / \gamma$. The ejecta now encounters more surrounding matter and decelerates faster than in the spherical case. The Lorentz factor now decays exponentially with the radius and as $\gamma \sim t^{-1 / 2}$ with observed time. Taking this into account, the observed break is even more significant. The slow cooling spectrum given in Figure 2 evolves now with decreasing peak flux $F_{\nu, m} \sim t^{-1}$, and the break frequencies evolve as $\nu_{m} \sim t^{-2}$, $\nu_{c} \sim t^{0}$ and $\nu_{a} \sim \ell^{-1 / 5}$. This translates to a temporal decay in a given frequency as listed in Table 1.

The jet break is a hydrodynamic one. It should therefore appear at the same time at all frequencies - an achromatic break. Though an achromatic break is considered to be a strong signature of a jet, onc should kecp in mind that any other

TABLE 1. The spectral index $\beta$ and the temporal index $\alpha$ as function of $p$ for a spherical and a jet-like evolution. Typical values are quoted using $p=2.4$. The parameter free relation between $\alpha$ and $\beta$ is given for each case (eliminating $p$ ). The difference in $\alpha$ between a jet and a sphere is always substantial at all frequencies.

\begin{tabular}{cccc}
\hline & $\begin{array}{c}\text { Spectral Index } \\
\beta, F_{\nu} \propto \nu^{-\beta}\end{array}$ & $\begin{array}{c}\text { Light Curve Index } \alpha, F_{\nu} \propto t^{-\alpha} \\
\text { sphere }\end{array}$ & jet \\
\hline \hline$\nu<\nu_{a}$ & $\beta=-2$ & $\alpha=-1 / 2$ & $\alpha=0$ \\
\hline$\nu_{a}<\nu<\nu_{m}$ & $\beta=-1 / 3$ & $\alpha=-1 / 2$ & $\alpha=1 / 3$ \\
\hline$\nu_{m}<\nu<\nu_{c}$ & $(p-1) / 2 \cong 0.7$ & $\alpha=3(p-1) / 4 \cong 1.05$ & $\alpha=p \cong 2.4$ \\
& & $\alpha-3 \beta / 2$ & $\alpha-2 \beta+1$ \\
\hline$\nu>\nu_{c}$ & $p / 2 \cong 1.2$ & $\alpha=(3 p-2) / 4 \cong 1.3$ & $\alpha=p \cong 2.4$ \\
& & $\alpha=3 \beta / 2-1 / 2$ & $\alpha=2 \beta$ \\
\hline
\end{tabular}


hydrodynamic transition will also produce an achromatic break. To name a few, the transition from relativistic to non-relativistic dynamics, a jump in the ambient density, or the supply of new energy from slower shells that catch up with the decelerated flow. However, the breaks produced by the transition from a spherical like evolution (when $1 / \gamma<\theta_{0}$ ) to a spreading jet has a well defined prediction for the change in the temporal decay indices. The amount of break depends on the spectral regime that is observed. It can be seen from Table 1 that the break is substantial $\Delta \alpha>0.5$ in all regimes and should be easily identified.

Finally we note that if jet's opening angle is of order unity, the total energy may still be about an order of magnitude lower than the isotropic estimate. However, in this case the break will be "hidden" as it will overlap the transition to nonrelativistic dynamics. It was suggested that this is the case for GRB 970508 [11]

\section{OBSERVATIONAL EVIDENCE FOR JETS}

Evidence of a break from a shallow to a steep powerlaw was first seen in GRB $990123[12,13]$. Unfortunately the break was observed only in one optical band while the infrared data were ambiguous. Yet, the strongest evidence for this burst being a jet does not come from this optical break but rather from radio observation$\mathrm{s}$, as explained below. A famous and exciting event this year was the first detection of a bright (9th magnitude) optical emission simultaneous with GRB 990123 [14]. Another new ingredient in GRB 990123 is a radio flare [15]. Contrary to previous afterglows, where the radio peaks after a few weeks and then decays slowly, this burst had a fast rising flare, peaking around a day and then decaying quickly. Sari and Piran [16] have shown that the bright optical flash and the radio flare are related. Within a day the emission from the adiabatically cooling ejecta that produced the 60 s optical flash shifts into the radio frequencies. Given this interpretation, the regular forward shock emission should have come later, on the usual few weeks timescale. The fact that this "usual" forward shock radio emission did not show up is in agreement with the interpretation of this burst as a "jet" which causes the emission to considerably weaken by the time the typical frequency $\nu_{m}$ arrives to radio frequencies.

GRB 990510 had a very clear break simultaneously in all optical bands and in radio $[17,18]$. In GRB 990123 and GRB 990510 the transition times were about 2.1 days and 1.2 days reducing the isotropic energy estimate by a factor of $\sim 200$ and $\sim 300$, respectively. The total energy is now well below a solar rest mass!

Sari, Piran \& Halpern [19] have noted that the observed decays in GRB afterglows that do not show a break are either of a shallow slope of $\sim t^{-1.2}$ or a very steep slope of $\sim t^{-2.1}$. They argued that the rapidly decaying bursts are those in which the ejecta was a narrow jet and the break in the light curve was before the earliest observation. Interestingly, evidence for jets are found when the inferred energy $E_{i s o}$ (which does not take jets into account) is the largest. This implies that jets may account for a considerable fraction of the wide luminosity distribution seen in 

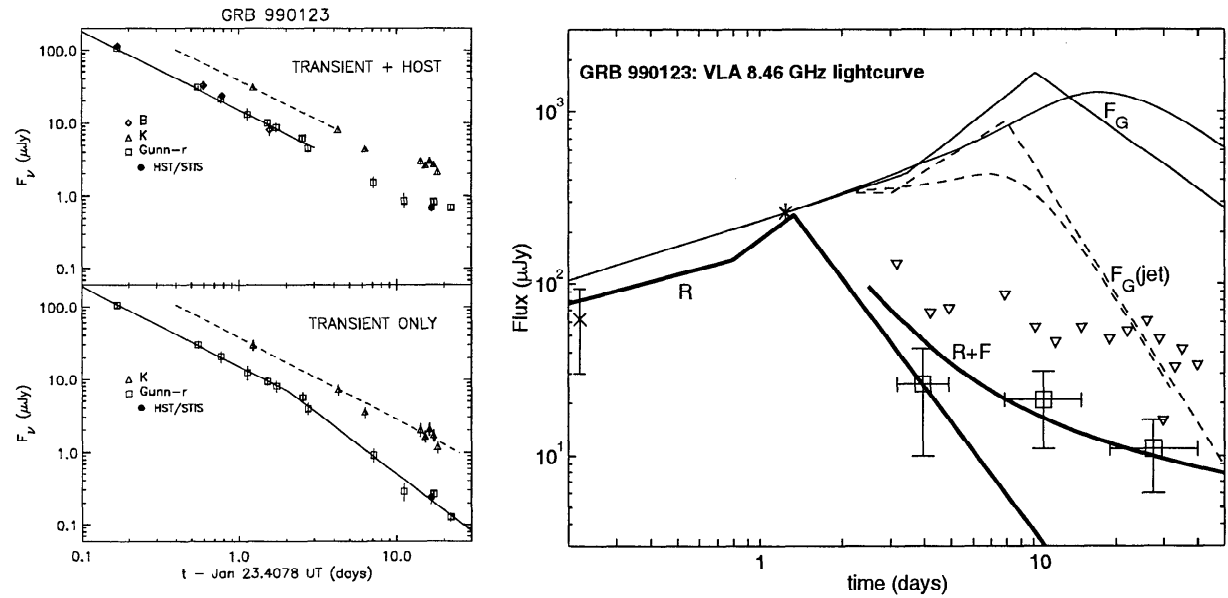

FIGURE 3. GRB990123: Optical data (left) shows some break in the light curve at Gunn-r band. $\mathrm{K}$ band seems to have no break but the contribution of the host galaxy is less certain. Radio "flare" (right) seen a day after the burst agrees with theoretical scaling of the optical flash (heavy solid line marked $\mathrm{R}$ ). In the jet interpretation, only faint radio emission is expected at late times as given by the heavy solid line marked $\mathrm{R}+\mathrm{F}$, in agreement with observations. Thin and dashed lines indicate the theoretical expectations if the radio signal at day two is interpreted as the forward shock (independent of the optical flash) and if jets are not taken into account. These will largely over predict the late radio upper limits [15], marked by triangles (see however [20]).
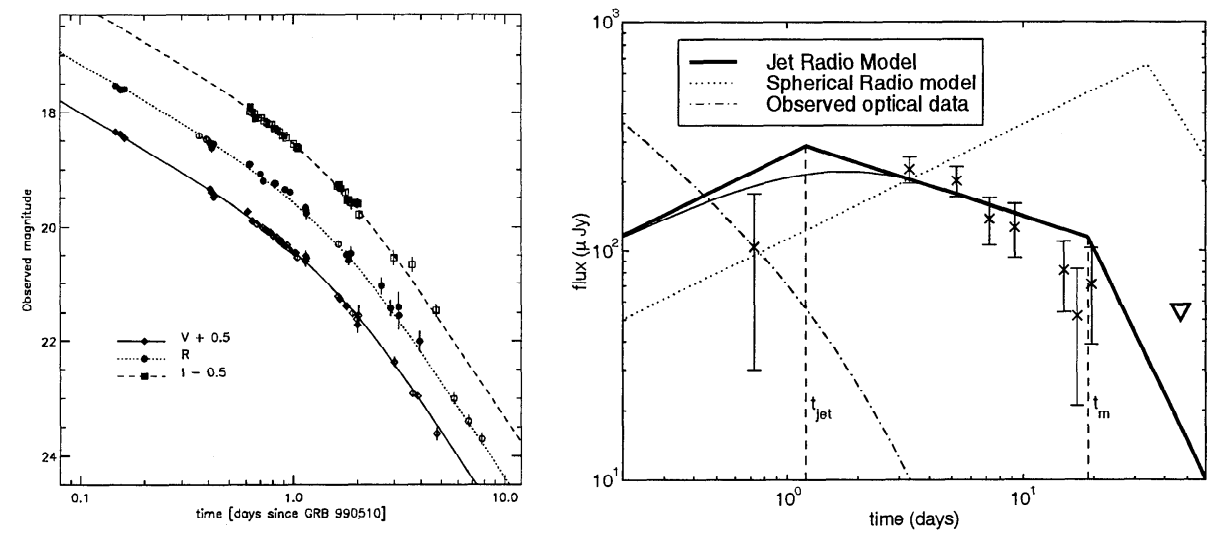

FIGURE 4. GRB 990510, the best evidence for a "jet": an achromatic break in optical and radio at $t_{\text {jet }} \cong 1.2$ days implying $\theta_{0} \cong 0.08$. The temporal slope beforc and after the break agrees woll with theory if $p \cong 2.2$. For this burst $E_{i s o}=2.9 \times 10^{53} \mathrm{erg}$ but the true total energy is only $E=10^{51} \mathrm{erg}$. 
GRBs, and that the true energy distribution is less wide than it seems to be.

An alternative explanation for these afterglows with fast decline is propagation into a medium with decreasing density, i.e., a wind produced earlier by the progenitor [21]. We favor the jet interpretation for two reasons: $(i)$ decreasing density only enhances the decay by $t^{-1 / 2}$ for $\nu_{m}<\nu<\nu_{c}$ and does not enhance the decay at all for $\nu>\nu_{c}$ (with typical parameters the optical and certainly the x-ray bands are above $\nu_{c}$ ). The rest of the needed effect, in the wind interpretation, is associated with a higher value of the electron powerlaw distribution index $p(p \cong 3$ instead of $p \cong 2.2-2.4$ ). Why should the value of $p$ be different for shocks propagating into winds? With the jet interpretation one can explain all afterglows with a single value of $p$, as in [19]. (ii) The jets interpretation makes the luminosity distribution of GRBs more narrow, since evidence for jets is found in bright events. Clearly, this is circumstantial evidence. A more clear cut between these two possible interpretations can be made with the use of early afterglow observations, preferably at radio frequencies (see [22]).

In summary, there are several kind of afterglows:

Shallow decline: $\sim t^{-1.2}$ for as long as the afterglow can be observed. These are probably spherical or at least have a large opening angle (e.g., GRB 970508).

Fast decline: $\sim t^{-2.1}$ (e.g., GRB 980519 and GRB 980326). These are either narrow jets, in which the break was very early, or they have high values of $p$ and propagate into decreasing density medium.

Breaks: Initially slow decline that changes into a fast decline. These are the best candidates for jets (e.g., GRB 990510).

\section{POLARIZATION - A PROMISING TOOL}

An exciting possibility to further constrain the models and obtain a more direct proof of the geometrical picture of "jets" is to measure linear polarization. High levels of linear polarization are usually the smoking gun of synchrotron radiation. The direction of the polarization is perpendicular to the magnetic field and can be as high as 70\%. Gruzinov \& Waxman [23] and Medvedev \& Locb [24] considered the emission from spherical ejecta by which symmetry should produce no polarization on the average, except for fluctuations of order of a few percent. Polarization is more natural if the ejecta is a "jet" and the line of sight from the observer is within the jet but does not coincide with its axis. In this case, the spherical symmetry is broken [25-27], and the natural polarization produced by synchrotron radiation should not vanish. For simplicity, let's assume that the magnetic field behind the shock is directed along the shock's plane (the results hold more generally, unless the magnetic field has no preferred direction). The synchrotron polarization from each part of the shock front, which is perpendicular to the magnetic field, is therefore directed radially.

As long as the relativistic beaming angle $1 / \gamma$ is narrower than the physical size of the jet $\theta_{0}$, one is able to see a full ring and therefore the radial polarization 

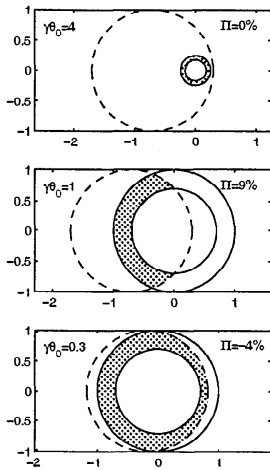
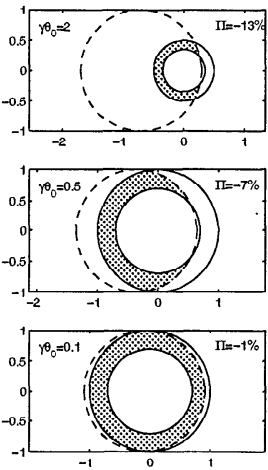

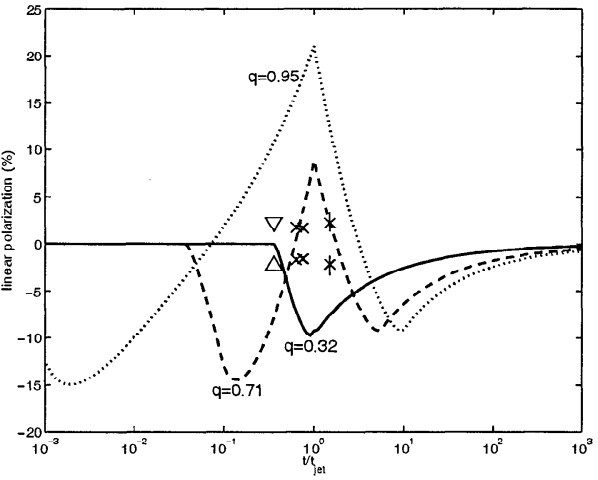

FIGURE 5. Left: Shape of the emitting region. Dash line marks the physical extent of the jet while solid lines give the vicwable region $1 / \gamma$. The obscrved radiation is coming from the gray region. On each frame, the percentage of polarization is given on the top right and the initial size of the jet relative to $1 / \gamma$ is given on the left. The frames are scaled so that the size of the jet is unity. Right: observed and theoretical polarization lightcurve for three possible offsets of the observer relative to the jet axis. Observational data for GRB 990510 is marked by $\mathrm{x}$, assuming $t_{j e t}=1.2 \mathrm{~d}$. The upper limit for GRB 990123 is given by a triangle, assuming $t_{j e t}=2.1 \mathrm{~d}$.

averages to zero (the first frame, with $\gamma \theta_{0}=4$ of the left plot in Figure 5). As the flow decelerates, the relativistic beaming $1 / \gamma$ becomes comparable to $\theta_{0}$ and only a part of the ring is visible; net polarization is then observed. Note that due to the radial direction of the polarization from each fluid element, the total polarization is maximal when a quarter $\left(\gamma \theta_{0}=2\right.$ in Figure 5$)$ or when three quarters $\left(\gamma \theta_{0}=1\right.$ in Figure 5) of the ring is missing (or radiate less efficiently) and vanishes for a full and for a half ring. The polarization when more than half of the ring is missing is perpendicular to the polarization direction when less than half of it is missing.

At late stages the jet expands and since the offset of the observer from the physical center of the jet is constant, spherical symmetry is regained. The vanishing and re-occurrence of significant parts of the ring results in a unique prediction: there should be three peaks of polarization, with the polarization position angle during the central peak rotated by $90^{\circ}$ with respect to the other two peaks. In case the observer is very close to the center, more than half of the ring is always observed, and therefore only a single direction of polarization is expected. A few possible polarization light curves are presented in Figure 5.

\section{SUMMARY}

Now when redshifts for GRBs are routinely measured, the largest uncertainty in their energy budget and event rate is the possibility that the emission is not spherical but jet-like. We discussed the theory of afterglow from jet-like event. 
These should produce a substantial break at all frequencies. The time where this break occurs is an indication of the jet's opening angle. GRB 990510 seems to be a perfect example of this behavior. The inferred opening angle is about 0.1 , consistent with upper limits from searches of orphan X-ray afterglows [28]. Several other candidates for jets are bursts with fast decline, where the break presumably took place before the earliest observation. This question will be settled when more frequent early observations are available. We have shown that afterglow from jets should show a unique signature of polarization at detectable levels. Observing such a signature will confirm the jet interpretation and the synchrotron model in general.

Acknowledgements. I thank Titus Galama for very useful comments, and the Sherman Fairchild foundation for support.

\section{REFERENCES}

1. MacFadyen, A. I., Woosley, S. E., ApJ 526, 152 (1999).

2. Sari, R., \& Piran T., ApJ 485, 270 (1997).

3. Fenimore, E. E., Madras, C., \& Nayakshine, S., ApJ 473, 998 (1996).

4. Piran, T., in the proceedings of the Gräftåvallen workshop "Gamma Ray Bursts: The First Three Minutes", ed. Juri Poutanen (1999).

5. Sari, R., Piran, T. \& Narayan, R., ApJL, 497, L17 (1998).

6. Galama, T. J. et al., ApJ 500, 101 (1998).

7. Rhoads, J. E., ApJ 525, 737 (1999).

8. Panaitescu, A. \& Mészáros, P., ApJ 503, 314 (1999).

9. Mészáros, P., \& Rees M. J., MNRAS 299, L10 (1999).

10. Moderski, R., Sikora, M., Bulik, T., astro-ph/9904310 (1999).

11. Frail, D. A., Waxman, E. \& Kulkarni, S. R., astro-ph/9910319 (1999).

12. Kulkarni, S. R. et al., Nature 398, 389 (1999).

13. Fruchtcr, $\Lambda$. S. ct al., $\Lambda p J$ 519, L13 (1999).

14. Akerlof, C. et al., Nature 398, 400 (1999).

15. Kulkarni, S. R. et al., ApJL 522, L97 (1999).

16. Sari, R., \& Piran T., ApJL 517, L109 (1999).

17. Stanek, K. Z., Garnavich, P. M., Kaluzny, J., Pych, W. \& Thompson, I., ApJL 522, L39 (1999).

18. Harrison F. A. el al., ApJL 523, L121 (1999).

19. Sari, R., Piran, T., \& Halpern, J. 1999, ApJ, 519, L17

20. Galama, T. J. et al., Nature 398, 394 (1999).

21. Chevalier, R. A. \& Li, Z. Y., $A p J$ in press, and astro-ph/9908272.

22. Frail, D. A. et al., astro-ph/9910060 (1999).

23. Gruzinov A., \& Waxman E., ApJ 511, 852 (1999).

24. Medvedev, M. V., \& Loeb A., astro-ph/9904363 (1999).

25. Gruzinov A., ApJL 525, L29 (1999).

26. Ghisellini, G., \& Lazzati, D., MNRAS 309, L7 (1999).

27. Sari, R., ApJL 524, L43 (1999).

28. Greiner, J. et al., these proceedings. 